\title{
Relation between homocysteine and biochemical bone turnover markers and bone mineral density in peri- and post- menopausal women
}

\author{
Markus Herrmann ${ }^{1, *}$, Marius Kraenzlin², \\ Gerhard Pape ${ }^{1}$, Marga Sand-Hill ${ }^{1}$ and Wolfgang \\ Herrmann ${ }^{1}$ \\ ${ }^{1}$ Abteilung für Klinische Chemie und \\ Laboratoriumsmedizin/Zentrallabor, \\ Universitätsklinikum des Saarlandes, Homburg/Saar, \\ Germany \\ ${ }^{2}$ Klinik für Endokrinologie, Diabetologie und \\ klinische Ernährung, Universitätsspital Basel, Basel, \\ Switzerland
}

\begin{abstract}
Background: Recently, increased plasma homocysteine (Hcy) has been suggested as an independent risk factor for osteoporotic fractures. Therefore, it is tempting to speculate that Hcy adversely affects bone metabolism. This study aimed to analyze the relation between Hcy and biochemical markers of bone metabolism and bone mineral density (BMD). Materials and methods: We investigated 143 peri- and post-menopausal women [median age (25th-75th percentile), 67 (57-75) years]. All subjects underwent a detailed medical examination, measurement of bone mineral density at lumbar spine (BMD-LS) and total hip (BMD-HIP), and fasting venous blood and urine sampling. Osteocalcin $(\mathrm{OC})$, serum calcium (Ca), urinary desoxypyridinoline cross-links (DPD), osteoprotegerin (OPG) and soluble receptor activator of NF$\kappa \mathrm{B}$ ligand (sRANKL) were studied. Results: According to BMD subjects were classified as normal $(n=24)$, osteopenic $(n=51)$ or osteoporotic $(n=68)$. Median Hcy did not differ between normal, osteopenic and osteoporotic subjects $(p=0.647)$. Partial correlation analysis, controlling for the major confounders, age, creatinine, menopause and previous fractures, revealed significant correlations between Hcy and DPD ( $r=0.193, p=0.022)$, as well as between Hcy and $\mathrm{Ca}(r=0.170, p=0.045)$. After adjustment for the same confounders, subsequent regression analysis confirmed significant associations of Hcy with DPD and Ca. No significant relations could be observed between Hcy and BMD-LS, BMD-HIP, OC, OPG or sRANKL. Conclusion: Our results demonstrate weak, but significant, relations between Hcy and markers of organic and inorganic bone resorption, suggesting a mechanistic role of Hcy in bone metabolism. The rela-
\end{abstract}

*Corresponding author: Dr. med. Markus Herrmann, Abteilung für Klinische Chemie und Laboratoriumsmedizin/ Zentrallabor, Universitätsklinikum des Saarland, 66421 Homburg/Saar, Germany

Phone: +49-6841-1630707, Fax: +49-6841-1630703,

E-mail: markusherr@aol.com tion between Hcy and bone resorption was not dependent on OPG or sRANKL.

Keywords: bone mineral density; bone turnover markers; cross-links; desoxypyridinoline; homocysteine; osteocalcin.

\section{Introduction}

Osteoporosis is a major public health problem, causing considerable morbidity and mortality (1-3). It has been estimated that in a 10 -year period, postmenopausal white women in the US will experience 5.2 million fractures of the hip, spine, or distal forearm, which will lead to 2 million person-years of fracturerelated disability and to over $\$ 45$ billion in direct medical expenditure (4). A comparable situation is evident in Europe $(5,6)$. Prevention of osteoporosis by identifying risk factors or risk indicators is therefore a major issue. Previous studies found advancing age, female gender, early menopause, low body weight, cigarette smoking, alcohol consumption, low calcium intake, a low physical activity level, tallness, prior lowtrauma fracture as an adult, and history of hip fracture in a first-degree relative as important risk factors for osteoporosis (7-9).

Recently, it has been suggested that an increased plasma homocysteine (Hcy) level is an independent risk factor for osteoporotic fractures in elderly persons $(10,11)$. Moreover, Hcy-lowering therapy with high doses of folate and vitamin $B_{12}$ has been shown to strongly reduce the hip fracture rate in stroke patients (12). However, all these studies are of epidemiological nature, providing no information about the mechanisms behind the relation between Hcy and fracture risk.

Previous investigations reported no or only weak associations between Hcy and bone mineral density (BMD) (11, 13-15). Since BMD measurement detects changes in bone metabolism with a significant delay (16-18) and provides no or only poor information about the microarchitecture of the bone matrix, these findings are not surprising. On the contrary, markers of bone resorption and formation have been proposed as a tool for the early detection of changes in bone metabolism $(19,20)$. Even if their clinical use is still a matter of debate (19), these markers could be a useful tool in demonstrating a relation between Hcy and bone metabolism. At present, few data exist regarding the relation between $\mathrm{Hcy}$ and biochemical bone turnover markers (21-23). Moreover, nothing is known about Hcy and the osteoprotegerin (OPG)/sol- 
uble receptor activator of NF-кB ligand (sRANKL) system, which is centrally involved in activation and differentiation of osteoclasts. Such data would provide a first indication of the existence of a mechanistic link between Hcy and bone metabolism and would help to clarify the role of hyperhomocysteinemia (HHcy) in osteoporosis.

We hypothesized that plasma $\mathrm{Hcy}$ is related to biochemical bone turnover markers and the OPG/ sRANKL system. Consequently, in this study we investigated Hcy, biochemical bone turnover markers, OPG, sRANKL and BMD in peri- and post-menopausal women.

\section{Materials and methods}

\section{Study design}

In this cross-sectional study we investigated 143 peri- and post-menopausal women with a median (25th-75th percentile) age of $67(57-75)$ years, a median height of 161 $(156-166) \mathrm{cm}$ and a median weight of $60(54-67) \mathrm{kg}$, who were occasionally recruited from an endocrinological clinic in Basel, Switzerland. To cover the whole range of bone loss, we selected individuals according to their BMD and tried to include sufficient numbers of normal, osteopenic and osteoporotic individuals (Table 1). None of the subjects was diagnosed with abnormal BMD (t-score at hip or lumbar spine $<-1.5)$ prior to the study. Consequently, none of the subjects was undergoing anti-osteoporotic treatment when blood was sampled. Subjects were excluded if they used pharmaceuticals known to interfere with bone metabolism (e.g., corticosteroids, anti-osteoporotic drugs, B vitamins).

All women were interviewed and examined by the same experienced endocrinologist, who was blinded to the Hcy measurement. For each subject, a detailed medical history, physical examination, BMD measurement at lumbar spine and total hip, venous blood sampling (after overnight fasting) and urine collection (first morning void) were carried out. Blood levels of Hcy, osteocalcin (OC), calcium (Ca) and creatinine, as well as urine levels of desoxypyridinoline cross-links (DPD) were measured. In addition, in a subset of 89 individuals, OPG and SRANKL were determined. Daily calcium intake was anamnestically assessed, and estimated on the basis of supplement use and milk consumption. Patient characteristics are summarized in Table 1. The study was conducted in accordance with the guidelines of the Decla- ration of Helsinki and was approved by the institutional Review Board.

\section{BMD measurement}

BMD at lumbar spine (BMD-LS) and hip (BMD-HIP) was evaluated by dual X-ray absorptiometry (DXA) using a Lunar Expert densitometer (24) (GE Healthcare, Freiburg, Germany). Lumbar vertebrae with prevalent or incident fractures at L1-L4 were not included in the BMD measurement. BMD was expressed as $\mathrm{g} / \mathrm{cm}^{2}$ of hydroxyapatite and as t-score for the respective anatomical site according to the European reference database of the Lunar Expert device.

\section{Pre-analytical sample handling}

Fasting venous blood samples were drawn during the office visits between 08:00 and 10:00 h. Samples were allowed to clot for $30 \mathrm{~min}$ before serum was separated from cellular blood components by centrifugation. Urine samples were obtained from the first morning void. Serum and urine samples were stored at $-20^{\circ} \mathrm{C}$ until analysis.

\section{Laboratory analysis}

Hcy was measured on an ADVIA CENTAUR automated analyzer (Bayer Diagnostics, Fernwald, Germany) using a commercial chemiluminescence immunoassay (Bayer Diagnostics). Intra- and inter-assay CVs were $\leq 5.2 \%$. OC was detected with a commercial chemiluminescence immunoassay (Roche Diagnostics, Mannheim, Germany) on an Elecsys 2010 automated analyzer (Roche Diagnostics). Intra- and inter-assay $\mathrm{CVs}$ were $\leq 7.2 \%$. Creatinine and calcium were measured using methods from Roche Diagnostics on a Hitachi 917 analyzer (Roche Diagnostics). OPG and sRANKL were analyzed by ELISA (Immundiagnostik, Bensheim, Germany). Intra- and inter-assay imprecision were $\leq 9.0 \%$ for sRANKL and $\leq 10 \%$ for OPG.

Urine DPD was measured with the Crosslinks ${ }^{\circledR}$ assay (BioRAD, Munich, Germany) by high-performance liquid chromatography (HPLC) and the results are expressed in relation to urinary creatinine. The detection limit was $5.0 \mathrm{pmol} / \mathrm{L}$, with a reference range of $9-20 \mathrm{pmol} / \mathrm{mmol} \mathrm{DPD} /$ creatinine. Urinary creatinine was assayed on a Hitachi 917 automated analyzer using a method from Roche Diagnostics. For quality control we used the appropriate quality control materials provided by the test manufacturers.

Table 1 Summary of anthropometric data and risk factors for osteoporosis.

\begin{tabular}{|c|c|c|c|c|}
\hline & All subjects & Normal BMD & Osteopenia & Osteoporosis \\
\hline Number & 143 & 24 & 51 & 68 \\
\hline Age, years & $67(57-75)$ & $55(50-67)$ & $68(60-70)^{*}$ & $71(61-77)^{*}$ \\
\hline Weight, kg & $60(54-67)$ & $64(58-62)$ & $63(56-70)$ & $58(53-63)^{* \S}$ \\
\hline Height, cm & $161(156-166)$ & 163 (160-167) & $161(156-166)$ & $160(154-165)^{*}$ \\
\hline BMI & $23(21-26)$ & $24(21-29)$ & $24(22-26)$ & $23(21-25)$ \\
\hline Peri-menopausal, n & 16 & 12 & 3 & 10 \\
\hline Post-menopausal, n & 127 & 12 & $48 *$ & $58^{*}$ \\
\hline Age at menopause, years & $49(45-50)$ & $54(47-56)$ & $49(48-51)$ & $48(45-50)$ \\
\hline Previous fractures, $\mathrm{n}$ & 37 & 1 & 3 & $33^{* \S}$ \\
\hline Daily calcium intake, mg & 769 (519-1049) & $901(621-1186)$ & $651(467-965)$ & $844(544-1117)$ \\
\hline Previous or current & 0 & 0 & 0 & 0 \\
\hline
\end{tabular}

vitamin use, $\mathrm{n}$

Normal BMD, t-score at hip and lumbar spine > -1.5 ; osteopenic, BMD t-score at hip or lumbar spine between - 1.5 and $-2.5)$; osteoporotic, BMD t-score at hip or lumbar spine $<-2.5$. ${ }^{*}$ vs. normal subjects, vs. osteopenic subjects. Values are given as median (25th-75th percentile). 


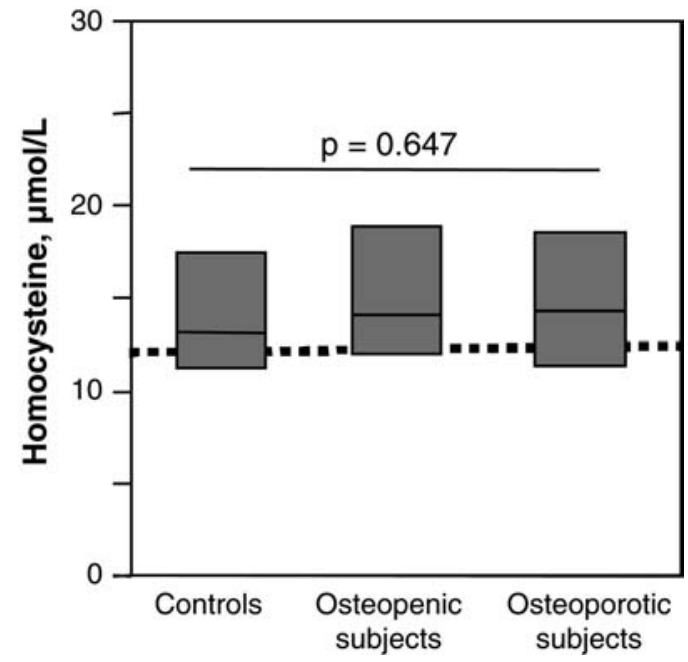

Figure 1 Boxplot (25th-75th percentile) showing the median $\mathrm{Hcy}(\mu \mathrm{mol} / \mathrm{L})$ concentration in normal (BMD t-score at hip and lumbar spine $>-1.5$ ), osteopenic (BMD t-score at hip or lumbar spine between -1.5 and -2.5 ) and osteoporotic (BMD t-score at hip or lumbar spine $<-2.5$ ) subjects. The dashed line shows Hcy cut-off of $12 \mu \mathrm{mol} / \mathrm{L}$.

\section{Statistical analysis}

First we performed a Kolmogorov-Smirnov test to check all variables for normality. Since several variables were not distributed normally, we report the descriptive statistics as median (25th-75th percentile). Medians were compared using a Mann-Whitney U-test. If more than two medians were compared, we used a Kruskal-Wallis test. First, we analyzed median Hcy levels in relation to diagnostic groups according to BMD (see Results). Subsequently, we performed a partial correlation analysis, controlling for the major confounders, age, creatinine, menopausal status (perior post-menopausal) and previous fractures. Since Hcy was not distributed normally ( $p=0.005$, Kolmogorov-Smirnov test), we log-transformed Hcy (log Hcy) prior to correlation analysis. Variables that were significantly correlated to log Hcy were then used as independent variables in a linear regression analysis, adjusting for the major confounders, age, creatinine, menopause and previous fractures. A value of $p<0.05$ was considered as significant. Calculations were carried out with the software package SPSS (version 11.0 for Windows; SPSS Inc., Chicago, IL, USA).

\section{Results}

According to BMD measurement, subjects were classified as normal (t-score at lumbar spine and hip $>-1.5 ; n=24$ ), osteopenic ( $t$-score at lumbar spine or hip between -1.5 and $-2.5 ; n=51$ ) or osteoporotic ( $t$ score at lumbar spine or hip $<-2.5 ; n=68$ ). The percentage of peri-menopausal women was highest in subjects with normal BMD (Table 1). As expected, median age increased with decreasing BMD. Furthermore, median weight and height decreased with decreasing BMD. Previous fractures were reported more frequently in osteoporotic than in osteopenic or normal women (Table 1).

Median Hcy did not differ between normal, osteopenic and osteoporotic individuals $(p=0.647$; Figure 1). Moreover, partial correlation analysis controlling for age, creatinine, previous fractures, and menopause did not reveal significant correlations between log Hcy and BMD-HIP $(r=0.104, p=0.234)$ and BMDLS $(r=0.157, p=0.070)$.

For the biochemical bone turnover markers, partial correlation analysis controlling for age, creatinine, previous fractures, and menopause revealed a significant relation between log Hcy and DPD ( $r=0.193$, $\mathrm{p}=0.022$; Figure 2). Serum $\mathrm{Ca}$, which is mainly released from the inorganic bone matrix, was also correlated to $\log \mathrm{Hcy}(r=0.170, p=0.045$; Figure 2). In contrast, OC, OPG and SRANKL exhibited no association with log Hcy. The adjusted regression model confirmed that log Hcy was independently related to DPD and Ca (Table 2).

\section{Discussion}

The main finding of this study is a weak, but significant, relation between Hcy and the bone resorption

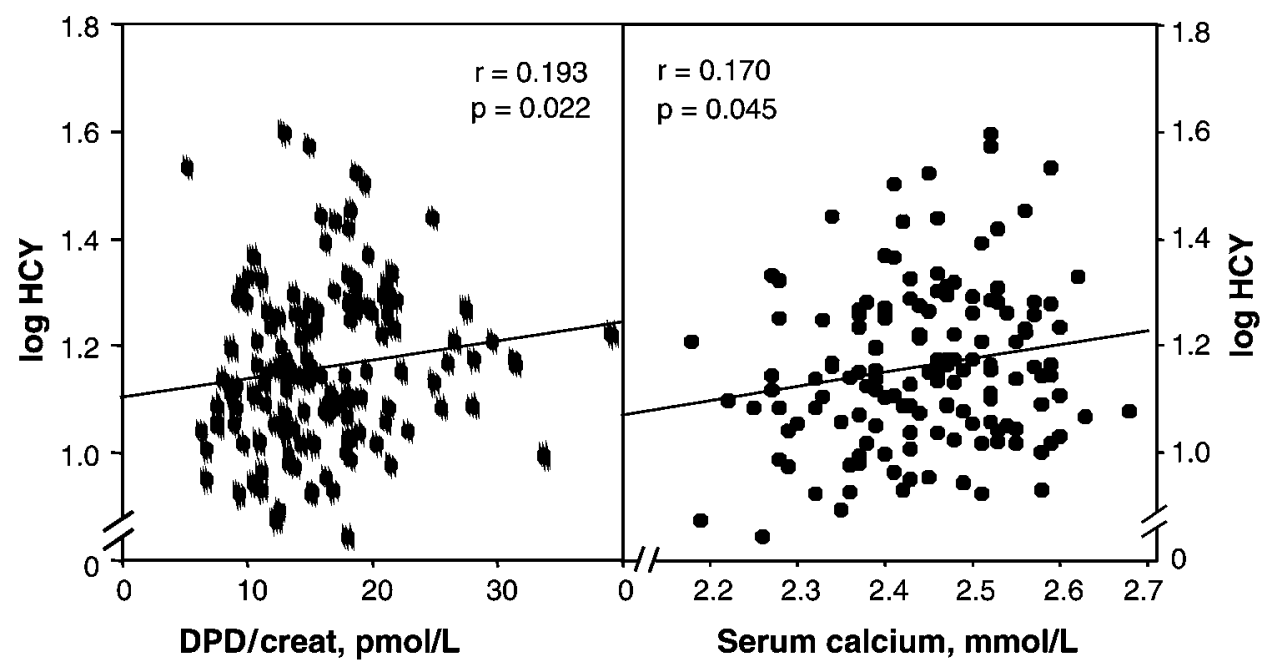

Figure 2 Partial correlation analysis between log Hcy and DPD and serum calcium, controlling for age, creatinine (creat), menopause, and previous fractures. 
Table 2 Regression analysis using DPD and Ca as dependent variables and log Hcy as independent variable.

\begin{tabular}{|c|c|c|c|}
\hline $\begin{array}{l}\text { Dependent } \\
\text { variable }\end{array}$ & Adjusted for & $\beta$ & $\mathrm{p}$ \\
\hline \multirow[t]{4}{*}{ DPD } & Raw & 5.33 & 0.105 \\
\hline & Age, creatinine, previous fractures, menopause & 8.22 & $0.022 *$ \\
\hline & Previous fractures, menopause, creatinine & 7.33 & $0.029 *$ \\
\hline & Previous fractures, creatinine & 6.22 & $0.050 *$ \\
\hline \multirow[t]{5}{*}{$\mathrm{Ca}$} & Raw & 0.12 & $0.037 *$ \\
\hline & Age, creatinine, previous fractures, menopause & 0.13 & $0.045^{*}$ \\
\hline & Age, previous fractures, creatinine & 0.13 & $0.037 *$ \\
\hline & Age, previous fractures & 0.14 & $0.017^{*}$ \\
\hline & Age & 0.12 & $0.037 *$ \\
\hline
\end{tabular}

$\beta$ - and $p$-values are presented for the raw model, as well as for several adjusted models, including the major confounders, age, creatinine, menopause and previous fractures. BMD-LS, bone mineral density at lumbar spine; DPD, urine cross-links; Ca, serum calcium. ${ }^{*} \mathrm{p}<0.05$.

marker DPD, suggesting a mechanistic link between Hcy and bone metabolism. This hypothesis is supported by the association between Hcy and serum $\mathrm{Ca}$. Contrary to DPD and $\mathrm{Ca}$, the bone formation marker $\mathrm{OC}$ and BMD at lumbar spine or hip were not associated with Hcy. Furthermore, higher DPD and Ca levels in the presence of elevated serum Hcy were not accompanied by differences in OPG or SRANKL, two central regulators of the equilibrium between osteoblasts and osteoclasts.

Osteoporosis is mainly diagnosed by BMD measurement and anamnestic data (25). In our sample, Hcy was not related to BMD-LS and BMD-HIP. This finding is in accordance with previous studies (11, 13-15) showing no or only a weak association of Hcy and BMD. However, the lack of correlation between Hcy and BMD is not surprising, since BMD mainly reflects bone mineralization and provides only an integral measure of bone metabolism over time $(16-18,26)$. BMD does not reflect the current status of bone metabolism and presents only poor information about the microarchitecture of bone matrix.

Biochemical markers of bone resorption and formation have been proposed as a tool for the early detection of changes in bone metabolism $(19,20)$ and might be more suitable for detecting the effects of Hcy on bone metabolism than BMD. The present study revealed weak, but significant, correlations between Hcy and the bone resorption marker DPD, as well as between Hcy and $\mathrm{Ca}$ (representing resorption of inorganic bone matrix), but no correlation between Hcy and the bone formation marker OC. These findings were still significant after adjustment for the major confounders, age, renal function, menopause and previous fractures, suggesting an association between Hcy and bone resorption activity. The relation between Hcy and DPD is in agreement with previous findings by others (21-23). Bode et al. reported a relation with comparable strength between Hcy and collagen I C-terminal cross-links, another well-established bone resorption marker, in patients with coronary artery disease (21). Miyao et al. found higher DPD levels in 57 subjects with a homozygous MTHFR C677T genotype, which is known to be associated with higher Hcy levels (22). Unfortunately, they did not measure Hcy, which limits the comparability with our results. Recent data from the Longitudinal Aging
Study Amsterdam (LASA) confirmed a significant correlation between Hcy, vitamin $B_{12}$ and DPD in 615 men and 652 women (23). Contrary to our results, a positive relation between Hcy and OC was also observed. However, a relation between Hcy and DPD and $\mathrm{OC}$ was only detectable in the presence of low vitamin $B_{12}$ levels, indicating an important mechanistic role of vitamin $B_{12}$ in bone metabolism.

In the study presented here, and in all of the abovementioned publications, the strength of the relations observed between Hcy and DPD, OC and $\mathrm{Ca}$ was relatively weak, suggesting that these results can probably not explain the impressive clinical results observed by others previously (10-12). McLean et al. demonstrated a notable increase in fracture risk at Hcy levels between 14 and $19 \mu \mathrm{mol} / \mathrm{L}$ (10). Based on our results, an increase from 10 to $15 \mu \mathrm{mol} / \mathrm{L}$ would only cause a marginal increase in DPD of approximately $1.5 \mathrm{pmol} / \mathrm{mmol}$. Similar results were found in all other studies reported (21-23). In view of the discrepancy between our results and the clinical data, it can be speculated that the stimulation of bone matrix degradation by Hcy is of limited clinical relevance and that use of the currently available bone turnover markers is not appropriate for monitoring the biochemical effects of Hcy or B-vitamin deficiencies on bone metabolism.

In patients with homocystinuria, characterized by extremely high Hcy levels and an increased prevalence of skeletal deformities, including osteoporosis (27-29), McKusick (30) proposed a disturbed crosslinking of collagen, which has been supported by two later studies $(31,32)$. Lubec et al. observed lower collagen I C-terminal telopeptide (ICTP) levels in 10 patients with homocystinuria, concluding that this would indicate disturbed collagen I cross-linking (32). However, the majority of the existing bone resorption markers, including DPD, measure the breakdown of collagen I (17) and detect constituents of the non-helical N- or C-terminal telopeptides, or the cross-linking molecules of collagen I. Based on clinical and analytical experience, DPD is currently considered the best marker for the assessment of bone resorption $(21,17)$. Contrary to bone resorption markers, OC is a small hydroxyapatite-binding protein mainly synthesized by osteoblasts (17). It is therefore considered as a relative specific marker of osteoblast function (17). How- 
ever, none of the existing biochemical bone turnover markers has been shown to provide information about the microstructure of bone matrix (e.g., crosslinking of collagen molecules, integration of collagen molecules in bone matrix, interconnection of spongy trabeculae), which is probably crucial for mechanical stability. Due to the severe limitations of the few studies available, McKusick's hypothesis of disturbed collagen cross-linking has to be considered as not proven. Moreover, it is not clear whether findings in patients with homocystinuria are directly applicable to normal Hcy variations among adults.

Nevertheless, together with existing studies, the present study provides a clear indication of the existence of a mechanistic role of Hcy in bone metabolism, and in particular in bone resorption. This conclusion is supported by cell culture experiments with human osteoclasts showing a dose-dependent increase in osteoclast activity with increasing Hcy concentrations in the culture medium (33).

OPG and SRANKL are central regulators of the equilibrium between osteoclast and osteoblast activity (34-37). In this study we did not observe an association between Hcy and OPG or SRANKL, indicating that Hcy does not affect bone metabolism by the OPG/ sRANKL system. However, the significance of this finding is limited by the relatively low sensitivity of the existing first-generation OPG and sRANKL immunoassays. Future studies should focus on free sRANKL, which possibly better detects modified OPG-binding capacity.

In conclusion, the present study demonstrates a weak relation between Hcy and the bone resorption marker DPD, as well as between Hcy and serum $\mathrm{Ca}$, suggesting a mechanistic link between Hcy and bone metabolism. However, the associations observed were not strong enough to explain the known relation between Hcy and fracture risk, and could not be supported by other biochemical markers of bone metabolism, such as OC, OPG or sRANKL. Therefore, future studies are needed to provide more insight into the mechanistic role of Hcy in osteoporosis as a useful paradigm with interesting therapeutic implications, because Hcy-lowering therapy could favorably influence the disease course in osteoporotic patients.

\section{References}

1. Ray NF, Chan JK, Thamer M, Melton LJ III. Medical expenditures for the treatment of osteoporotic fractures in the United States in 1995: report from the National Osteoporosis Foundation. J Bone Miner Res 1997;12: 24-35.

2. Melton LJ III. Adverse outcomes of osteoporotic fractures in the general population. J Bone Miner Res 2003; 18:1139-41.

3. Center JR, Nguyen TV, Schneider D, Sambrook PN, Eisman JA. Mortality after all major types of osteoporotic fracture in men and women: an observational study. Lancet 1999;353:878-82.

4. Chrischilles E, Shireman T, Wallace R. Costs and health effects of osteoporotic fractures. Bone 1994;15:377-86.
5. Kanis JA, Jonsson B. Economic evaluation of interventions for osteoporosis. Osteoporos Int 2002;13:765-7.

6. Walker-Bone K, Walter G, Cooper C. Recent developments in the epidemiology of osteoporosis. Curr Opin Rheumatol 2002;14:411-5.

7. Kanis JA, Black D, Cooper C, Dargent P, Dawson-Hughes B, De Laet C, et al. A new approach to the development of assessment guidelines for osteoporosis. Osteoporos Int 2002;13:527-36.

8. Eisman J, Clapham S, Kehoe L. Osteoporosis prevalence and levels of treatment in primary care: the Australian BoneCare Study. J Bone Miner Res 2004;19:1969-75.

9. Hodgson SF, Watts NB, Bilezikian JP, Clarke BL, Gray TK, Harris DW, et al. American Association of Clinical Endocrinologists medical guidelines for clinical practice for the prevention and treatment of postmenopausal osteoporosis: 2001 edition, with selected updates for 2003. Endocr Pract 2003;9:544-64.

10. McLean RR, Jacques PF, Selhub J, Tucker KL, Samelson EJ, Broe KE, et al. Homocysteine as a predictive factor for hip fracture in older persons. $N$ Engl $\mathrm{J}$ Med 2004;350:2042-9.

11. van Meurs JB, Dhonukshe-Rutten RA, Pluijm SM, van der Klift $\mathrm{M}$, de Jonge $\mathrm{R}$, Lindemans $\mathrm{J}$, et al. Homocysteine levels and the risk of osteoporotic fracture. N Engl J Med 2004;350:2033-41.

12. Sato $Y$, Honda $Y$, Iwamoto J, Kanoko T, Satoh K. Effect of folate and mecobalamin on hip fractures in patients with stroke: a randomized controlled trial. J Am Med Assoc 2005;293:1082-8.

13. Cagnacci A, Baldassari F, Rivolta G, Arangino S, Volpe A. Relation of homocysteine, folate, and vitamin B12 to bone mineral density of postmenopausal women. Bone 2003;33:956-9.

14. Golbahar J, Hamidi A, Aminzadeh MA, Omrani GR. Association of plasma folate, plasma total homocysteine, but not methylenetetrahydrofolate reductase C667T polymorphism, with bone mineral density in postmenopausal Iranian women: a cross-sectional study. Bone 2004;35:760-5.

15. Morris MS, Jacques PF, Selhub J. Relation between homocysteine and B-vitamin status indicators and bone mineral density in older Americans. Bone 2005;37: 234-42.

16. Lenchik L, Kiebzak GM, Blunt BA. What is the role of serial bone mineral density measurements in patient management? J Clin Densitom 2002;5(Suppl):S29-38.

17. Bonnick SL. Current controversies in bone densitometry. Curr Opin Rheumatol 2002;14:416-20.

18. Deal CL. Using bone densitometry to monitor therapy in treating osteoporosis: pros and cons. Curr Rheumatol Rep 2001;3:233-9.

19. Delmas PD, Eastell R, Garnero P, Seibel MJ, Stepan J. The use of biochemical markers of bone turnover in osteoporosis. Committee of Scientific Advisors of the International Osteoporosis Foundation. Osteoporos Int 2000;11(Suppl 6):S2-17.

20. Stepan JJ. Prediction of bone loss in postmenopausal women. Osteoporos Int 2000;11(Suppl 6):S45-54.

21. Bode MK, Laitinen $P$, Risteli J, Uusimaa $P$, Juvonen $T$. Atherosclerosis, type 1 collagen cross-linking and homocysteine. Atherosclerosis 2000;152:531-2.

22. Miyao M, Morita $H$, Hosoi T, Kurihara $H$, Inoue $S$, Hoshino $S$, et al. Association of methylenetetrahydrofolate reductase (MTHFR) polymorphism with bone mineral density in postmenopausal Japanese women. Calcif Tissue Int 2000;66:190-4.

23. Dhonukshe-Rutten RA, Pluijm SM, de Groot LC, Lips P, Smit JH, van Staveren WA. Homocysteine and vitamin B12 status relate to bone turnover markers, broadband ultrasound attenuation, and fractures in healthy elderly people. J Bone Miner Res 2005;20:921-9. 
24. Njeh CF, Apple K, Temperton DH, Boivin CM. Radiological assessment of a new bone densitometer - the Lunar EXPERT. Br J Radiol 1996;69:335-40.

25. Meunier PJ, Delmas PD, Eastell R, McClung MR, Papapoulos S, Rizzoli R, et al. Diagnosis and management of osteoporosis in postmenopausal women: clinical guidelines. International Committee for Osteoporosis Clinical Guidelines. Clin Ther 1999;21:1025-44.

26. Cummings SR, Bates D, Black DM. Clinical use of bone densitometry: scientific review. J Am Med Assoc 2002;288:1889-97.

27. Grieco AJ. Homocystinuria: pathogenetic mechanisms. Am J Med Sci 1977;273:120-32.

28. Morreels CL Jr, Fletcher BD, Weilbaecher RG, Dorst JP. The roentgenographic features of homocystinuria. Radiology 1968;90:1150-8.

29. Mudd SH, Skovby F, Levy HL, Pettigrew KD, Wilcken B, Pyeritz RE, et al. The natural history of homocystinuria due to cystathionine beta-synthase deficiency. Am J Hum Genet 1985;37:1-31.

30. McKusick V. Heritable disorders of connective tissue, 3rd ed. St Louis, MO: Mosby, 2004.
31. Kang AH, Trelstad RL. A collagen defect in homocystinuria. J Clin Invest 1973;52:2571-8.

32. Lubec B, Fang-Kircher S, Lubec T, Blom HJ, Boers GH. Evidence for McKusick's hypothesis of deficient collagen cross-linking in patients with homocystinuria. Biochim Biophys Acta 1996;1315:159-62.

33. Herrmann M, Widmann T, Colaianni G, Colucci S, Zallone A, Herrmann W. Increased osteoclast activity in the presence of elevated homocysteine levels. Clin Chem. In press.

34. Hofbauer LC, Kuhne CA, Viereck V. The OPG/RANKL/ RANK system in metabolic bone diseases. J Musculoskelet Neuronal Interact 2004;4:268-75.

35. Hofbauer LC, Schoppet M. Clinical implications of the osteoprotegerin/RANKL/RANK system for bone and vascular diseases. J Am Med Assoc 2004;292:490-5.

36. Schoppet M, Preissner KT, Hofbauer LC. RANK ligand and osteoprotegerin: paracrine regulators of bone metabolism and vascular function. Arterioscler Thromb Vasc Biol 2002;22:549-53.

37. Khosla S. Minireview: the OPG/RANKL/RANK system. Endocrinology 2001;142:5050-5. 